\title{
THE ORIGIN AND GROWTH OF MATHEMATICAL CONCEPTS
}

\author{
R. L. WILDER
}

1. Introduction. According to A. N. Whitehead [1, p. 20], "The science of Pure Mathematics, in its modern developments, may claim to be the most original creation of the human spirit"; a statement with which probably few mathematicians would quarrel. A layman, however, might and probably would take this to mean that modern mathematics is something which has already been created; a creation, that is, which has already been accomplished and is now safely embalmed with remains on view in any good library.

Of course, as mathematicians we know that mathematics is in no such static shape; that it is, on the contrary, a dynamic affair, changing even from day to day. Our late colleagues who were in the forefront of mathematical creation about the turn of the century, 1900, would be amazed to see what mathematics is like today; indeed, many of them, I'd wager, would not like some of the modern developments, probably taking the same attitude toward them that some mathematicians of the late 19th century took toward Cantor's innovations regarding the infinite. Moreover, those of us who are active today would probably feel the same way toward the mathematics of the year 2000, if we were in some way able to view it. We ourselves probably have not sufficient perception of the changes going on in mathematics at the present time; it is well known that the participants in great social changes are usually unaware of them. And there is some evidence that our awareness of the process of mathematical change, although not so at variance with the facts as that of the layman, is still so defective in some ways as to lead to unfortunate but avoidable situations. This is partly due, I suppose, to our being so busy creating new mathematics that we have little time or patience to view our behavior from the outside and study its characteristics. And even when we do so, we seem prone to take such a specialized angle from which to make our observations that we get only a partial perspective.

2. Motives. Poincaré remarked, in one of his numerous essays [2, p. 376], that "mathematical science must reflect on itself," and

A retiring address, as Vice President and Chairman of Section A, American Association for the Advancement of Science, delivered before a joint meeting of Section A, the American Mathematical Society, and the Mathematical Association of America, at St. Louis, Missouri, December 29, 1952; received by the editors March 13, 1953. 
supported this dictum with several studies of the psychological aspects of mathematical creation. These studies inspired others of a similar nature, among which might be noted especially the little book [3] by Hadamard on the Psychology of invention in the mathematical field, published 7 years ago.

However, the angle from which these studies were made, that of psychology, can furnish only part of the general picture. Studies of a psychological nature are significant in that they analyze the mental processes by which the individual mathematician uses the materials and tools present in his culture to make new constructs. But they fail to make proper connection with the cultural stream in which they are imbedded. The mathematician is not an isolated entity grinding out new ideas, with everything coming out and nothing going in. Rather he is making new syntheses out of the concepts that are going in. Young men who have just received the doctorate usually seem to have at least an intuitive recognition of this, for how often do we hear them express their fears of having to accept positions where there are few or no mathematicians who have interests similar to their own; or, if they find it necessary to accept such positions, they worry over whether the libraries, by which they mean the means of keeping contact with their fellow workers through the medium of journals, are good or not. The popularity of such centers as Göttingen in the twenties, and now the Institute for Advanced Study, furnishes further evidence of the gregariousness of the mathematician. And of course there are our societies and associations, through whose meetings and journals we may exchange ideas.

I believe, therefore, that even if it only leads to a rational understanding and appreciation of these matters, a study of these cooperative features of mathematical creation, or what we might call a study of the "group dynamics" of mathematical creation, is warranted. Because of its high level of abstraction, we are inclined to look upon mathematics as strictly an individual activity. In his address opening the recent International Congress of Mathematicians in Cambridge [4, p. 125], Oswald Veblen comments that "Mathematics is terribly individual. Any mathematical act, whether of creation or apprehension, takes place in the deepest recesses of the individual mind." However, he goes on to say, "Mathematical thoughts must nevertheless be communicated to other individuals and assimilated into the body of general knowledge. Otherwise they can hardly be said to exist." Note that last sentence, by the way"Otherwise they can hardly be said to exist." Obviously Veblen is aware that while, in one of its aspects, mathematical creation is, as 
he says, "terribly individual," in its other aspects it is not an individual affair at all. Later, in the same address, we find an affirmation of the necessity for mathematicians forming such associations as we have today: "The resultant organizations of various kinds have accomplished many important things known to us all. Of these accomplishments I am sure that the most important is the maintenance of a set of standards and traditions which enable us to preserve that coherent and growing something which we call Mathematics." [Italics mine.]

To summarize my motives, then, I wish to inquire, above the individual level, into the manner in which mathematical concepts originate, and to study those factors that encourage their formation and influence their growth. I think that much benefit might be derived from such an inquiry. For example, if the individual working mathematician understands that when a concept is about to make its appearance, it is most likely to do so through the medium of more than one creative mathematician; and if, furthermore, he knows the reasons for this phenomenon, then we can expect less indulgence in bad feelings and suspicion of plagiarism to ensue than we find in notable past instances. Mathematical history contains numerous cases of arguments over priority, with nothing settled after the smoke of battle has cleared away except that when you come right down to it practically the same thing was thought of by someone else several years previously, only he didn't quite realize the full significance of what he had, or did not have the good luck to possess the tools wherewith to exploit it. Coolidge, in his $A$ history of geometrical methods, remarks [5, p. 122], "It is a curious fact in the history of mathematics that discoveries of the greatest importance were made simultaneously by different men of genius." This is quite true, except that there is nothing "curious" about it, nor is it confined to mathematics. And it is exactly what one should expect if he is acquainted with the manner in which concepts evolve.

I shall have to be brief and incomplete in my presentation here, giving only a general description of my study. In particular, I shall pay little attention to questions such as "What is a concept?", or to the different type levels of concepts, important though these are. I shall take it for granted that we know what we mean by such terms as "the group concept," "the concept of limit," and the like-these are well defined today. Other things which we label "concepts" are not at all well defined. And of course some concepts embrace other concepts, such as that of the calculus embracing the concept of limit of a set of real numbers. 
Furthermore, in the case histories which I shall presently mention, you will probably be impressed with the fact that in tracing the roots of a concept under discussion, we find invariably that some of them go back to Greece. It should not be inferred from this that they began in Greece. The fact is, that these roots have many smaller tendrils that reach out into pre-Greek or contemporary cultures, and I am not enough of a historian to have their identities, if such exist, at my disposal. This will not, however, affect my main conclusions in the least. I do want to warn, however, against the impression that these roots necessarily start in Greece.

In reviewing such case histories, I am of course going over much material which is well known. What we get out of such material, however, is strongly influenced by our orientation, and in repeating these historical details it is with the purpose of bringing out aspects that are not ordinarily emphasized and frequently not observed.

3. Case studies. Now how do mathematical concepts originate; where do they come from? I suppose we might, without thinking very seriously about our answer, reply, "By taking thought;" or "by shutting one's self in his study, getting out a pencil or piece of chalk, and going to work." This kind of reply is reminiscent of the answer which someone $^{1}$ made to the question, "How does one write novels?", viz., "By pressing the seat of one's pants to a chair for three hours at a time and writing."

Like most pat answers, this is not, on further reflection, quite acceptable. To be sure, concepts don't get born without someone taking thought. We must not forget, however, that gregarious aspect of mathematical activity which I mentioned above. Not only does this have its influence on the formation of concepts, but it must be conceded an equal partner, at least, in the process.

A concept doesn't just pop up full grown "like Venus from the waves," although it may seem to, to the individual mathematician who does the conceiving. Usually its elements are lying in what, if the term were not already in technical mathematical use, one might call the mathematical continuum, but which we might better call the mathematical culture stream. As the eminent historian of science, George Sarton, comments [6, p. 36]: "-creations absolutely de novo are very rare, if they occur at all; most novelties are only novel combinations of old elements, and the degree of novelty is thus a matter of interpretation, which may vary considerably according to the historian's experience, standpoint, or prejudices. - the determina-

\footnotetext{
${ }^{1}$ I think it was the writer Sinclair Lewis, but I have been unable to confirm this.
} 
tion of an event as the 'first' is not a special affirmation relative to that event, but a general negative proposition relative to an undetermined number of unknown events."

3a. Number and geometry concepts. The absurdity of always striving for a point of origin is nowhere better exemplified than in the origins of mathematics itself. We are unable here to find any "names" by which to label innovations even if we would. And this, I am certain, is not due simply to absence of adequate written record. The accompanying figure is supposed to represent the origin and growth of the earliest mathematical concepts, those of number and geometry.

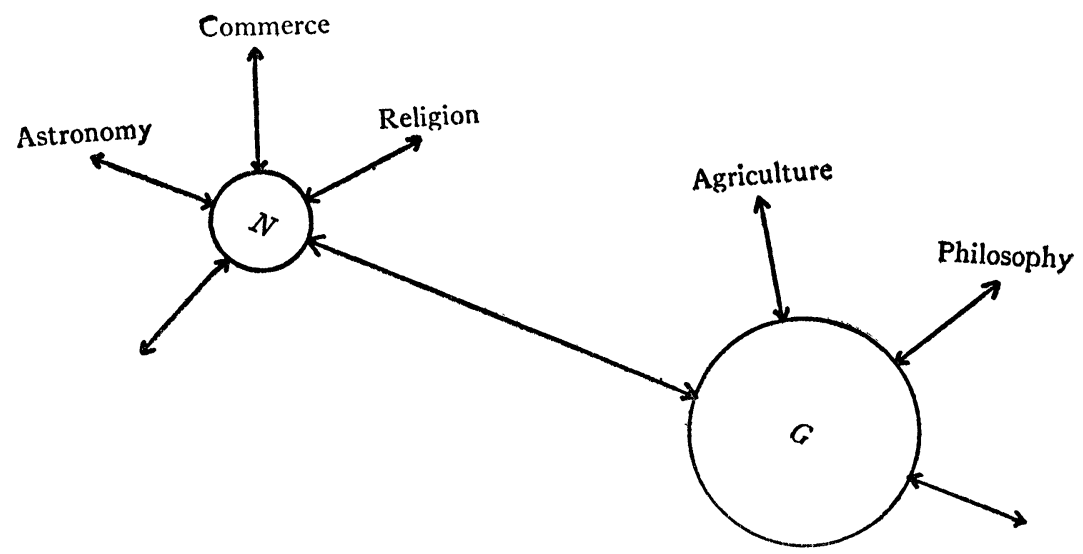

Just when these began to merit being called concepts we don't know, but enough evidence exists to warrant the conclusion that this occurred at no particular date, since each gradually emerged from the cultures of the Middle East and Greece; and of course they may have emerged in other cultures, as the number concept did in the Mayan culture. The short arrows represent such influences as those of a Babylonian priesthood facing the necessity of assessing taxes and keeping records of a host of tax-gatherers, the desire to compute the quantity of seeds necessary to sow a given piece of land, and other social needs. From such matters to the concept of a number-"four" for example, as contrasted with "four men," "four dogs"-must have been a long evolution. Certainly no one has suggested a prehistoric Archimedes leaping out of his bath or primitive swimming pool and running through the forest primeval shouting, "I've got it-the number four, the number four!" Similarly, geometry did not begin with Euclid.

3b. Analytic geometry. Or to take a very elementary, but his- 
torically important example, in histories of mathematics one finds serious discussion of whether Fermat or Descartes should be credited with the "invention" of analytic geometry, sometimes accompanied by a choice between the two and an extended argument supporting the choice.

In such cases, I am inclined to feel that the historian is unconsciously influenced by that principle of popular folklore; "everything has a beginning." And conceding this, no historian worth his salt can fail to produce one. In some cases, particularly in that of a very specialized concept, such as that of the "theta-Fuchsian series" whose genesis in the mind of Poincaré is so interestingly described in his article on Mathematical creation [2, p. 387], to speak of a "beginning" is perhaps justified-although even in such instances one could without doubt, given sufficient evidence, trace the inception of the notion from prior concepts. But in the case of broader concepts, such as "analytic geometry," we seem prone to error due to the vagueness of the concept.

It is interesting to note the way in which Coolidge handles the question in his $A$ history of geometrical methods. To quote [5, p. 117]: "The opinion is currently held among mathematicians that analytic geometry sprang full-armed from the head of Descartes as did Athene from that of Zeus.... There is much to be said in favor of this thesis, but . . . another opinion is certainly possible. The fact is that in inquiring into the origin of analytic geometry we run into a difficulty that lies at the bottom of a good proportion of our disputes in this Vale of Tears. What do we mean by the words 'analytic geometry'? Till that is settled, it is futile to inquire as to who discovered it." That the essence of the subject, however we define it, was known to the Greeks, such as Apollonius, seems beyond dispute; but as a coordinate geometry with a workable algebra like that which we associate with the notion, it evolved through the works of Fermat and Descartes as well as various predecessors. Had the Greeks possessed a good algebra, the story might have been different.

3c. Calculus. Everyone is familiar with the way the basic postulate of popular folklore that everything has a beginning has operated in the case of the Calculus. The dispute over its so-called "authorship" between Newton and Leibniz and their followers is, I suppose, the classical example of useless disputation attributable to the impulse to conform to the postulate. In a recent article [7], A. Rosenthal makes clear the indebtedness of both Newton and Leibniz to their forerunners as well as their contemporaries; and that no matter how you define it, the calculus is a product of a slow evolution that has been 
recorded as far back as the Greeks. And although I suspect that the author privately feels that it makes little sense to speak of the invention or the discovery of the calculus as an actual temporal event, he tries to justify the thesis that Newton and Leibniz originated the concept, by affording a definition.

After remarking that "there certainly was an extensive development of the theory of integration and differentiation in the period immediately before Newton and Leibniz," he asks [7, p. 83], "What then was missing at that time," -i.e., at the time when Newton and Leibniz entered the picture. He points out that one "very important point still missing was the general fact that differentiation and integration are inverse processes, that is, the so called fundamental theorem of integral calculus." And after some discussion as to whether various mathematicians, who evidently came very close to it, really recognized it or not, he discloses that it was known in full generality by Isaac Barrow, the teacher of Newton at Cambridge. So he repeats, "What more remained to be done?" replying, "What had to be created was just the Calculus, a general symbolic and systematic method of analytic operations, to be performed by strictly formal rules, independent of the geometric meaning. . . . it is just this Calculus which was established by Newton and Leibniz, independent of each other and using different types of symbolism." Although this is not really a definition in the true sense, at least it is an attempt to fix the general nature of just what it was that Newton and Leibniz did do. Whether it is a good thing to try to assign an originator to as broad a concept as the calculus, even when it is impossible to make the originator unique, is another question.

It should be noted that, as in the case of analytic geometry, if the Greeks had possessed enough symbolic material, they might have progressed further with the calculus. Also that despite the lack of a rigorous theory of limits, that which most agree to call "calculus" appeared nevertheless with Leibniz and Newton, but essentially as an operational apparatus. The theory of limits, and calculus as we know it, had still to evolve.

It is interesting to compare Coolidge's comments [5, p. 38]. He speaks of the Greek geometers' "procedure in attacking problems in computation which led naturally to the integral calculus. If we seek the root of the difference between their method of attack and ours, it lies partly in the greatly increased flexibility of our notation, partly in their lack of a clear concept of a limit." That is, a suitable notation was lacking - a suitable tool-and a necessary concept, that of limit, had yet to evolve. Since the concept of limit was not developed until 
after the work of Newton and Leibniz, this seems to place the emphasis again upon the Greek lack of an algebraic tool, as the root of the difference between the Greek calculus and that of Leibniz and Newton.

3d. The curve concept. Finally, I would like to view the evolution of a narrower, more specialized concept, that had its origin in antiquity, and which not only continued its growth in modern times but contributed in a noteworthy manner to a large portion of modern mathematics. It is not, I am sure, generally realized how great an influence this concept has had on the development of concepts which today contain hardly a trace of the notion. I refer to the concept of curve.

The accompanying chart, which may be referred to during the discussion, is supposed to set forth the principal lines of influence, and it is to these that I shall confine myself. It would be impossible to sketch here the history of the concept in full detail. It has been a common mathematical notion, as a rule purely intuitive, since the time of the Greeks, at least. And it is one of those mathematical notions that have been adopted by the layman. Usually it has been just a conventional categorical term, and seems to have continued so throughout the time when the study of curves was resumed analytically during the Renaissance. The first successful attempts at formulating precise definitions of the concept were not made by geometers, but by analysts! So far as I have been able to find, Georg Cantor was the first to be credited with a definition, but apparently what he really did $[8$, p. 194$]$ was to determine those settheoretic properties that constitute the continuous, as opposed to the discrete, aspect of a curve-or as a topologist would express it, he defined the topological notion of continuum. But a square together with its interior is a continuum, and we would hardly call this a curve! However, if the continuum lies in the plane, all we need to do is require that it contain no such square and we get what came to be called a "cantorian line." And although satisfactory only as a definition of the concept of plane curves, the cantorian line coincides exactly with what most topologists feel is the natural definition.

The next most notable attempt at definition was also not made by a geometer, but by C. Jordan in 1887. In the first (1882) volume of his Cours d'analyse [9], he speaks (as on p. 238, loc. cit.) of a plane curve as given by any two functions $x=f(t), y=g(t)$, where the range of $t$ is the real number interval $[0,1]$. Although no mention of continuity is made, if he was familiar with Cantor's proof $[8$, p. $122 \mathrm{ff}$.] published in 1878, 4 years earlier, of the (1-1)-correspondence be- 


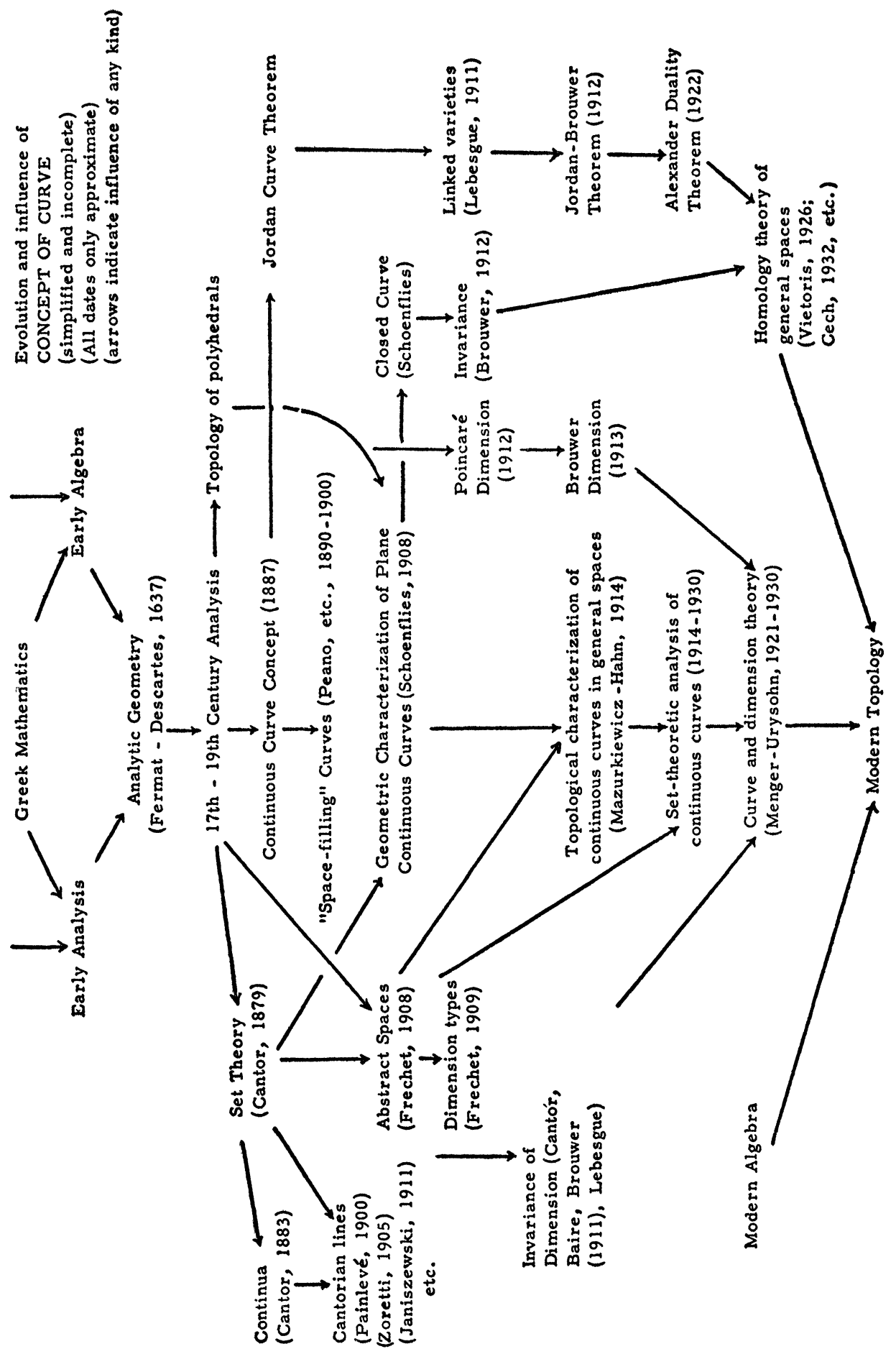


tween the points of such an interval and the points of the square plus its interior, he must have realized that without it, this definition could not be satisfactory. However, in an appendix ("Note") to the 3rd volume of this work, published 5 years later, in repeating this definition, he added $[9$, vol. 3, p. 587], "If the functions are continuous, the curve will be called continuous." (See also [9a, p. 92]; this reference to the first volume of the second edition, 1893, is the one customarily cited as the "origin" of the concept "continuous curve.") Cantor had noted that the (1-1)-correspondence just referred to was impossible in case the functions were continuous, and Jordan's definition of continuous curve lay between the two extremes-it does not require (1-1)-ness, although the functions are of course single-valued, and it does require continuity.

It is not difficult to surmise where Jordan got this notion; it was common practice to define curves parametrically in the books on calculus. For instance, the parametric representation of the cycloid is given in Cauchy's famous calculus [10, p. 52], published in 1826. Evidently what Jordan did was to formulate a general concept which was already current. I don't know who first attached Jordan's name to the notion, as a matter of fact. The Polish topologists were already doing so during the 'teens of the present century, and most writers of the twenties assume he was the first to formulate it explicitly. However, I have found no evidence that either Jordan's contemporaries or his immediate successors associated his name with the concept, which seems to be further evidence that the notion was generally current among analysts.

But to get on with the story: Geometers, possibly becoming suddenly conscious of their inadequacy, or perhaps jealous of their prerogatives, pounced upon the notion. I am confident that Jordan had no idea of usurping their natural rights; that all he had in mind was to make precise certain analytic notions that were already current, for his own special purposes. For instance, he showed that for a curve to be rectifiable, it is necessary and sufficient that the functions defining it be of bounded variation [9, vol. 3, p. $594 \mathrm{ff}$.]. And for the proof of his famous theorem, the Jordan Curve Theorem [9, vol. 3, p. $587 \mathrm{ff}$.; 9a, p. 92], he stipulated that the continuous curve was to have no double points except at the end points of the interval of definition of the variable $t$. For these purposes, his definition was a good one, and still is.

As is well known, it was only three years after Jordan's formulation until the Italian logician and geometer Peano published, in 1890, his famous space-filling curve. Hilbert and E. H. Moore followed suit 
soon after (for discussion and references, see [13, p. $943 \mathrm{ff}$.]). None of them mentions Jordan's name, incidentally,-perhaps further evidence that the concept was probably not considered as his personal property! Their examples show, of course, that Jordan's definition was not a satisfactory formulation of the precise notion of curve as it was intuitively conceived.

However, fortunately for the development of modern mathematics, the fact that the geometer found Jordan's analytic definition wanting did not mean that it was doomed to oblivion so far as geometry was concerned; even though it came to be recognized that in addition to including configurations we don't want to call "curves," it excluded some we do want to call curves (such as the well known continuum formed from $\sin 1 / x$ and its limit points on the $y$-axis). That it included among its special cases the general euclidean space element-for of course the cube and its interior in three and higher dimensions are continuous curves in Jordan's sense-was of intense geometric interest. Thus it developed that interest in the continuous curve was focused on the nature of its geometric counterpart; if this counterpart was not what we call "curve" in geometry, just what configurations do come within its scope?

The first systematic investigation of this question was made by Schoenflies, who gave a complete answer for the planar continuous curves (for discussion and reference, see [13, p. $964 \mathrm{ff}$.]). His work, incidentally, shows a remarkable perception of the nature of the problem as well as ingenuity in selecting the right methods for solving it. Realizing that it could not be solved by any of the methods common to the older forms of geometry, he called upon the new set-theoretic ideas introduced by Cantor and the (apparently unrelated) topology of polyhedrals of Riemann and Poincaré.

Among the first to be influenced by Schoenflies' work was Brouwer, probably best known for his Intuitionism, but among topologists and analysts famous for the remarkable work which he did on the theory of manifolds, especially as regards mapping and fixed point theorems, during the second decade of the present century. Brouwer was impressed not only by Schoenflies' positive results, but by certain fundamental errors which, due to faulty intuition, subtly crept into Schoenflies' work. For in his study of the Jordan Curve Theorem, Schoenflies conceived the idea of separating, from its tie-up to the topological images of the circle, the property of a curve being "common boundary of two domains in the plane" and called any configuration having this property a "closed curve." That a closed curve so defined could not be the common boundary of more than two domains seemed 
perfectly natural to assume. Only it happens not to be true, as Brouwer was able to prove (for discussions and references, see [13, p. $924 \mathrm{ff}$.]). Also, Schoenflies was unable to give any proof of the invariance of the closed curve under topological transformations. Brouwer also supplied this [11], using a method of proof which contained the central idea of the extension, to general metric spaces, of the homology theory of polyhedrals which had been initiated by Riemann, Poincaré, and others, and extended in this country by Veblen and his colleagues.

But I must not get too far ahead. The threads of influence leading from Jordan's definition begin now to entangle with one another and with threads from other sources. About the time when Brouwer was writing his dissertation on the law of the excluded middle, with perhaps little thought of topology, Fréchet was working on his dissertation [12] on "abstract spaces." This tendency to abstract from the euclidean situation, which of course goes back, in its roots, to Fréchet's predecessors but was given clear-cut realization by him, was to influence the treatment of the continuous curve problem in the following manner: It had proved impossible, with the tools available at the time, to extend Schoenflies' methods to 3-space or higher dimensions. Here the point of view is relative to the imbedding space, the Euclidean space of 3 or higher dimensions, rather than intrinsic. The introduction of the abstract space idea led to the problem of intrinsic characterization, by geometric methods, of the continuous curve. In order to eliminate the separate functions defining the coordinates in Jordan's definition, one recognized that an equivalent idea is to consider the curve as defined by a single function, $f(t)$, single-valued and continuous over the interval $[0,1]$, but the values of the function to be in any space allowing of the definition of limit. From this point of view the problem became manageable and received a solution, independently, at the hands of Hahn and Mazurkiewicz. Using the topological notion of local connectedness, which had actually been introduced (presumably unknown to them) three years earlier by Pia Nalli in another connection (for reference, see [13, p. 922, footnote 224]), they characterized continuous curves as compact metric continua that are locally connected (for discussion and references, see [13, p. $947 \mathrm{ff}$.] - - a thoroughly intrinsic definition, as contrasted with the results of Schoenflies.

This was about the time of the start of World War I, and the simultaneity of these results did not become known until after the War. There followed, not only in Poland and Austria, but in this country where the students of E. H. Moore had become interested in 
the new ideas in the foundations of geometry, a fairly complete analysis, especially during the period up to 1930, of the concept of continuous curve from the set-theoretic point of view.

Now it is characteristic of mathematical concepts that they often tend to be swallowed, as for instance in the process of generalization, by more embracing concepts. As a rule, this happens subsequent to the formulation and investigation of the concept. The number concept is a simple example of this. But in the case of the curve concept, the assimilation was to be coincident with the formulation of the larger concept. And so, for a few minutes, I shall turn to the concept of dimension. I cannot take time to review its ancient historyremarks analogous to those which I made concerning the concept of curve hold here, such as its use by the Greeks, its adoption into popular parlance, etc. As we approach modern times, attempts to formulate a dimension concept begin to crop out in the literature, often in the works of analysts, whose formulation was usually based on the number of parameters involved in the description. Cantor gave the body blow to this when he showed [8, p. $122 \mathrm{ff}$.] that a single real parameter suffices, if one neglects continuity. The resulting investigations - and these included the Jordan formulation of curve as well as the Peano results already mentioned-especially the problem of the invariance of dimension under bicontinuous (1-1)-transformations, are too detailed for me to go into here. They played their part, however, in the evolution of the dimension concept. On the left-hand side of the chart will be found some indications of the miscellaneous work done in this direction - note the names of Baire, Brouwer, and Lebesgue accompanied by the date 1911. In addition certain dimension definitions or attempts at definitions are also indicated, and there is a notation regarding "cantorian lines," which were studied by both Zoretti and Janiszewski among others. (For references, see sections 12,17 of [13], especially pp. 907 and 951-952.)

Now I do not maintain that any particular mathematician read any of the work of these men, and was directly influenced thereby. I call attention to their work chiefly as a proof that the notions of curve (or "line" as it was frequently called) and dimension were evolving in the mathematical culture stream.

To the right of the center of the chart you will notice the notation "Poincaré dimension, 1912." It seemed appropriate to place this over to the other side of the chart for several reasons; the most important being that it leads quite naturally to the concept which was finally generally accepted as embodying the precise formulation of the intuitive notion of curve and dimension. Another reason for setting 
this apart from the material on the left side of the chart is that although Poincaré did the most important kind of work in laying the foundations of the topology of polyhedrals, as is well known he had little acquaintance with or use for the theory of sets. Consequently, when he tried to indicate just what the dimension of a space is, in such essays as that on The notion of space, for instance [2, p. $243 \mathrm{ff}$.] (or see [14, p. 3]), he thought globally instead of in the small. And so his definition is an "in the large" definition-which may suffice for a homogeneous space, but most topological spaces are not homogeneous. However, the terminology in which it is formulated, such as "continua" and "separating" or "cutting," were actually set-theoretic. As a result, the definition was not given in precise mathematical terms. Nevertheless, anyone who happened to notice it and who had the suitable tools in hand could make it precise and a better approximation to a satisfactory definition. And this is exactly what Brouwer did the very next year, in 1913. (See [14, p. 4].)

Brouwer's paper attracted little attention, and when, in the early twenties, a satisfactory theory of curve and dimension was announced by Menger and Urysohn (citations may be found in [15, p. $83 \mathrm{ff}$.]), evidently neither of these gentlemen knew of the work of Brouwer, nor of each other's work. It is now known, of course, that all three theories are equivalent for a wide class of spaces-the locally connected, separable metric spaces.

The arrows on the chart, which, as the key in the upper right-hand corner states, are supposed to represent influence of any kind, need perhaps some further explanation. That the curve and dimension theory of Menger and Urysohn was influenced by the various settheoretic activities of their predecessors is, I think, too obvious to need further comment. As I have already pointed out, it is probable that lack of contact with these could have prevented Poincare from setting up some kind of a precise definition of dimension. The arrows coming in from the sides, however, are not so obvious. Just how much, if any, of the works on dimension problems which preceded them were actually read by Menger or Urysohn, I don't know. In the introduction to his formal presentation of his work published in 1925, Urysohn refers to the work of Zoretti on cantorian lines, for instance, and not only adopts the same term but entitles his monographs Sur les multiplicités Cantoriennes; he also remarks that his dimension definition is quite in conformity with the desires that motivated Poincaré. But even if no such direct and obvious influences existed, there is still the indirect influence that may have been exercised through the medium of others. Menger frequently made gracious acknowledgment to his 
teacher, Hans Hahn, not, so far as I know, for specific suggestions regarding definitions but for general guidance and inspiration; Urysohn's association with Alexandroff is well known.

But my time is running out, and I cannot give further details. I have already mentioned how the Brouwer proof of invariance of the Schoenflies closed curve contained the germ of the application of the homology theory of polyhedrals to general spaces-a fact which led Vietoris, who acknowledges his debt to Brouwer, to extend this theory to general metric spaces in order to establish an intrinsic form of the analogous higher dimensional invariance. (For citations and sketch of the developments discussed here, see $[16$, p. 14].) Let me also call attention to the arrows on the extreme right of the chart leading from the Jordan Curve Theorem to work of Lebesgue and Brouwer in higher dimensions, and thence to the Alexander Duality Theorem (which was motivated by the desire to obtain a general higher-dimensional extension of the Jordan-Brouwer separation theorem), and hence into algebraic aspects of modern topology. Incidentally, I have said very little about the role played by algebra in these matters; but its influence is mainly quite modern, and my intent has been not to sketch a history of curve theory, but to indicate as well as I can, in the time at my disposal, the manner in which the curve concept has influenced various other concepts right down to the inception of modern topology. Of course other concepts played a part too, but as I said before the threads of influence become quite tangled, and it is better to follow one at a time.

Before leaving the chart, let me point out, to prevent misunderstanding, the "simplified and incomplete" notation in the upper right-hand corner. Many things have been omitted, not because they are unknown (although there are many such), but to avoid complicating the diagram. There are, for example, geometric contacts that are omitted. If only in the way of providing certain perspective, geometric concepts exercised an influence on set theory, for example, and on curve and dimension theory.

4. Some inferences from case histories. Now what can be inferred from such observations as these? We can hardly prove anything, in the sense that we prove a mathematical theorem, any more than we can prove that certain methods of teaching mathematics are the best we might use. In such matters we are dealing with a kind of "metamathematics," although not in the technical sense in which the term is being used, to be sure. We can, however, make certain inferences of value.

4a. Factors influential in concept formation. For the sake of com- 
pleteness we first note the existence of evolution of mathematical concepts from the general cultural environment-the non-mathematical environment, that is. This was not only operative in the origin of the primitive concepts concerning number and geometry, but has continued throughout mathematical history. It is going on today, as anyone who has worked on war or government projects can bear witness. And although it deserves closer study, I shall not give it any more attention here, but shall pass on to factors operative within mathematics. So from now on, in my conclusions, I shall treat mathematics as an organism which, although influenced by an outside environment, is going to be of interest only from the standpoint of its internal structure.

I have just used the term "organism." This was to emphasize the cooperative character of mathematical creation, as contrasted with the individual aspect. As Veblen observed, mathematics is "terribly individual." But we don't work in a vacuum. Neither, it is true, do we work in teams like the experimental scientists do, although we do collaborate in small groups of two or three-rarely in greater number. I have in mind, however, also those types of cooperation which are not so obvious-especially the type of cooperation I imply when I make the statement that a Newton can carry on only from the level which the mathematics of his time has reached. This is not peculiar to mathematics, of course-Beethoven was as much indebted to the musical developments of his predecessors as to the more obvious inventions of the musical scale in which he wrote and the instruments for which he composed. But I think that we mathematicians have not been properly conscious of the fact. When, for example, a genius of the stature of Leibniz expends so much energy in collecting evidence of the priority of his ideas over those of Newton (see [17], for instance), is it not clear that he is not properly aware of the debt owed to those on whose shoulders he stood? Or, when a concept has germinated and gradually emerged over a period of anywhere from 50 to 2000 years, and then is suddenly brought into full light through the inventive powers of two, or possibly three or four mathematicians -and when, moreover, historical research shows that others were nearly ready to bring out the idea-does it make sense to call the event a coincidence?

This is not to deprive brilliant intellects that make final syntheses of concepts of any credit. Without them, concepts would never get born. But it is equally true that without the cooperation which they have received from innumerable other workers, many of whom may be gone and forgotten, the material from which they made their 
syntheses, and the tools they used, would not have been available. And by properly giving credit where credit is due, perhaps more modesty in the effort to establish priority will make everyone concerned a little happier.

Of course many mathematicians have sensed the organic character of mathematics. One of the most recent examples I have noticed of this was in an article by $\mathrm{H}$. Weyl entitled $A$ half-century of mathematics [18]. When half-way through this article he pauses and exclaims, "The constructions of the mathematical mind are at the same time free and necessary. The individual mathematician feels free to define his notions and to set up his axioms as he pleases. But the question is, will he get his fellow-mathematicians interested in the constructs of his imagination? We can not help feeling that certain mathematical structures which have evolved through the combined efforts of the mathematical community bear the stamp of a necessity not affected by the accidents of their historical birth. Everybody who looks at the spectacle of modern algebra will be struck by this complementarity of freedom and necessity."

The individual mathematician cannot do otherwise than preserve his contact with the mathematical culture stream; he is not only limited by the state of its development and the tools which it has brought forth, but he must accommodate his desires to those concepts that have reached a state where they are ready for synthesis.

Analytic geometry could not be synthesized in the manner which Fermat and Descartes are usually credited with doing, until both the necessary geometry and algebra were at hand; nor until their predecessors had carried the synthesis nearly to fruition. The same remark holds for calculus. If we interpret the achievement of Newton and Leibniz as the creation of a symbolic method which synthesized those ideas of the calculus which were already in existence, then their dependence on the cooperation of others is clear. And it seems doubtful that Menger and Urysohn would have, independently, arrived at the precise notions of dimension and curve which they formulated, without the preliminary development of not only the topological tools necessary for their formulation, but of such concepts as that of localization which were not, apparently, possessed by Poincaré. And that a concept of dimension was trying to break through to the mathematical consciousness is evidenced by the related problems that had been treated during the preceding decades, as well as by its ultimate formulation by two independent workers (as in the case of analytic geometry and calculus).

As I said before, let me emphasize that I do not make these ob- 
servations with any intent of taking away credit where credit is due. Furthermore, we must beware of looking backward from the heights which we have now scaled and concluding that the tasks of our predecessors were easy. Not long ago I overheard a group of young topologists discussing the state of topology about 1920, particularly the fertile field for research which was opening up at that time in the investigation of continuous curves. They seemed quite in agreement that they had been born 30 years too late - that it is a much harder task to find a subject for a dissertation now than it was then. As I was one of those who had been born 30 years sooner, I felt compelled to point out what the situation really was like at that time. I could tell them, for instance, of one young mathematician, who had just received the $\mathrm{Ph}$.D. degree, and who decided to forsake topology since it was obviously all worked out! None of us really knew of the possibilities at that time. And much energy had to be expended on the invention of concepts which now seem almost trivial, and of new methods of attack.

The greatest factor in the evolution of concepts today is probably what I would call conceptual contacts; on the individual level, it is what we might call a "meeting of minds"; on the group level, it is the diffusion of concepts. Examples of the former, the meeting of individual minds, are to be found in the many cases of mathematicians, who, due to the political conditions prevailing in Europe the past 40 years, moved to new mathematical centers and established contacts with men whose ideas and methods often found fruitful syntheses with their own. Similar contacts are, of course, being made today through the medium of grants to foreign mathematicians enabling them to visit mathematical centers in this country-as well as in the reverse process wherein members of our group visit abroad. Diffusion of concepts on the group level is of that type which was exemplified in the past by the fusion of algebra and geometry to form analytic geometry; or by the diffusion into geometry of set-theoretic methods in order to provide satisfactory formulations of the curve and dimension concepts; or in the recent past by such fusions as those of algebra and topology to form algebraic topology or topological algebra, depending on which aspect of the fusion you place emphasis. Concepts such as these are not the result of the chance meeting of two mathematicians at a mathematical gathering or because of an invited lectureship, as was the case in some instances which I can recall; but resulted from the gradual building up, by many independent workers, of a host of component concepts. The manner in which such syntheses as these are brought about needs, I imagine, no 
further amplification. For the individual mathematical discipline they form the analogue, within the field of mathematics, of the interplay between mathematics and its environment in the general culture. They point up, of course, the desirability and the necessity, if we would encourage healthy mathematical growth, of inter-university cooperation on a more enduring basis than is provided by the meetings of societies; even though the latter do foster individual contacts and undoubtedly play an important part in developments on the higher group level.

Not far removed from the types of concept formation already mentioned is that which consists in the observation of similar patterns in several different branches of mathematics, or, at a lower level, in several special cases. The classic example is probably the concept of abstract group. The Jordan definition of continuous curve illustrates the formulation of a concept from observation of its special cases commonly used in analysis. And again this is a kind of conceptformation which, within mathematics, is the analogue of the type of concept-formation which has for its basis the observation of mathematical patterns in the external environment, particularly in physics.

Also of a related character is the introduction of new tools from other branches of mathematics. The concept of the general continuous curve space, formulated independently by Hahn and Mazurkiewicz, making use of the tools provided by abstract space concepts, is one example. Another example is the introduction of the axiomatic method into algebra with results which have been remarked upon by a number of writers, particularly Weyl [18]. A current example is the use of metamathematical tools to establish theorems about the theorems in a given branch or branches of mathematics.

This reminds one of certain methods, which, because of their wide applicability, have earned the right to special notice as conceptbuilders. The axiomatic method is one of the most notable of these. I cannot resist recalling here what Poincaré, in his article entitled The future of mathematics, conceived as the chief use to which the method would be put [2, p. 382]. He devoted less than half a page to it; remember that when he wrote, the method was still quite new in its modern sense. After commenting that Hilbert had obtained "the most brilliant results" with it, he observed that the problem of providing axiomatic foundations for various parts of mathematics would be very "restricted," and "there would be nothing more to do when the inventory should be ended, which could not take long. But when," he continued, "we shall have enumerated all, there will be many ways of classifying all; a good librarian always finds something to do, and 
each new classification will be instructive for the philosopher." It would seem that Poincaré believed that the method was of little importance for mathematical creation. I wonder what his opinion of it would be today, if he could see what a tool for research it has become?

Related to the axiomatic method, in that it provides a special field for its use, is the process of altering of concepts already formulated. This is the kind of thing we do when we replace an axiom, such as the parallel axiom of euclidean geometry for instance, by a contradictory-in the case mentioned replacing the parallel axiom by one of the types of non-parallel axioms. Here one has to be especially careful, of course, to preserve contact with established theories-or as Weyl put it, mindful of whether one will "get his fellow-mathematicians interested in the constructs of his imagination."

That this process operates on the group level as well as on the individual level is shown by the case already cited, that of non-euclidean geometry. When this began to break through to the individual mathematical consciousness, it had already smoldered for years in the fires of Saccheri's and others' determination to prove its logical impossibility. When it did find expression, it found it in several places. As Coolidge remarks [5, p. 73], "The outstanding effect of a comparison of the work of Lobachevski and Bolyai is surprise at their likeness. Both start with the parallel angle, both note the Euclidean nature of the geometry on the limiting surface, both develop the formula for corresponding lengths on parallel limiting surfaces, both note the relation to spherical trigonometry and the independence of this from the parallel axiom." And then when Gauss revealed that he had obtained almost identical results earlier, is it to be wondered that Bolyai suspected Lobachevski of plagiarism and exclaimed, "It can hardly be possible that two, even perhaps three, persons, knowing nothing of one another, have achieved almost complete the same results at about the same time, even if by different paths." It is a pity that he could not know that not only was it possible, but quite probable, since the evolution can be clearly traced in the mathematical and philosophical thought of the preceding centuries.

Then there is the well known and much abused generalization! That this operates on the individual level needs not to be elaborated on, since we all use it in our individual research. However, it does operate on the group level. For instance, that kind of generalization which results from the splitting of concepts may take place on the group level; I suppose the splitting of the concept of the real number system into its structural or topological aspects, and its operational or algebraic aspects, furnishes an example of this. Splitting of con- 
cepts, that is, may be due to the gradual evolution of the mathematical organism rather than to the conscious act of an individual mathematician-even though the final syntheses may be the acts of individuals.

4b. Life span of a concept. Having discussed some of the modes by which concepts originate, let us turn for a moment to the life of the concept, following its exact formulation. The examples of the precise formulation of the topological character of continuous curve by Hahn and Mazurkiewicz, and that of the general curve and dimension theory by Menger and Urysohn, are instructive here. It seems that the formulation of a new concept may result in a distinct branch, or sub-branch of mathematics as the case maybe-a kind of "specialty," we might call it. This may become so popular that one is not in the "swing" in his particular field unless he joins in the investigation of the new concept. Mathematics is as subject to fashions as any other aspect of man's behaviour! Sooner or later, however, the so-called specialty is developed to such an extent that either it loses its popularity or achieves immortality through contact with other concepts. Thus we find in the cases mentioned that as soon as the related widespread investigations had resulted in solutions of the main problems, the fundamental parts of the theories were either absorbed in new concepts or made part of the general framework, both as to tools and materials, of general topology. The theory of continuous curves contributed not only such notions as that of local connectedness and its properties to the study of other theories, such as that of Lie groups, but was absorbed in a new concept, that of $1 c^{n}$ spaces (see $[16, \mathrm{VI}]$, for instance), whose definition became possible because of the extension of homology tools to general spaces in the decade succeeding the investigations on continuous curves. Like comments can be made concerning dimension theory, whose fundamental notions are now part of the general equipment of a topologist; and which was absorbed in a more general theory of dimensions utilizing homological notions $[19 ; 14$, VIII $]$, and is constantly used in applications of topology. I presume that a more elementary example may be furnished by elementary geometry as the Greeks knew it. I imagine that it had already reached its zenith and was on the wane when Euclid wrote his encyclopaedic work-the historians can correct me if I am wrong about this. Here, if ever, was a case of a mathematical theory being embalmed. When mathematical archeologists, so to speak, resurrected it, it seems to have stimulated only futile attempts to prove the parallel postulate or to trisect the angle and square the circle. Only new perspective that comes with conceptual 
contacts and new tools could infuse life into it. And, of course, this came in the realization of the validity of the non-euclidean geometries, and the introduction of methods of algebra and analysis which resulted in the modern form of analytic geometry, and a surge upward in analysis following the rise of the calculus.

To summarize, then, a concept, having only a limited range of possibilities, seems to have a certain life span like that of a star, going through a period of tremendous activity and then waning. Without the infusion of, or fusion with, new conceptual material, it is probably destined to die. But there is insufficient evidence to judge of this, since fortunately the new material is usually ready. New tools may be introduced from other parts of mathematics or even from the same branch, or even suggested by cultural elements outside of mathematics; or the concept may join with other concepts to form new ones more fruitful than the old; I have mentioned examples of all these possibilities. I cannot, as a matter of fact, cite offhand any case of a mathematical concept that has died in the sense that it no longer has any use whatsoever. The accumulated body of knowledge to which it has led is sooner or later seized by workers in other branches of mathematics, or even outside of mathematics, and put to work-often in a way of which its devotees never dreamed.

An interesting fact, which is probably a result of this, is that whereas there may seem to emerge from all this the spawning of a host of narrow specialties, a process of unification is continually going on. It is as though mathematics strikes out in all visible directions, but conscious that it will never do to become too diverse, pauses periodically to consolidate and unify the gains made before making new advances. (For further discussion and case histories see [20] and citations therein.)

5. Import to the individual mathematician. Since I have spent so much time in this talk on the group level, I should like to make a comment or two about the implications to the individual. Some of these I mentioned in my introduction and will not repeat here. I should like to make one amplification of my previous remarks concerning the benefits that may accrue to the individual research worker, especially those who are starting their careers. I have been continually impressed, since beginning this study, with the manner in which it brings out not only the advisability and necessity of keeping in touch with the mathematical culture stream through the media of journals and personal contacts-something we already habitually do-but with the suggestive ideas that may be found by exploring the works of the past. I am not advocating here a mathe- 
matical "great books program," but just a little browsing now and then in the original attempts at formulations of commonly used concepts, as well as of concepts not yet satisfactorily defined. They often contain suggestions which were impossible of fulfillment at the time when written, because the tools for exploiting them were not available. It is not difficult to imagine that the dimension theory of Poincaré, which was published in a philosophical journal, might, if mathematics had developed in different fashion, not yet be susceptible to precise formulation; and would, in that case, be lying ready for the moment to arrive when the suitable tools become available.

For a similar reason, it seems that every new generation of mathematicians should give attention to the famous unsolved problemsfor one never knows when the tools adequate for a solution may have evolved.

6. Future developments. It is time I brought this talk to a close. In doing so, I hope you will be so kind as to let me indulge in a little speculation. Up to this point I believe I have stuck to facts as well as one can in a domain where, as I said before, proofs are not possible in the mathematical sense. What I want to say now I shall freely label "speculation," and you can take it for what it seems worth to you.

Whenever I reflect on the changes that have occurred in mathematics during the past 40 years, I invariably recall that statement of Spengler whose Decline of the West, published in 1918, caused such a commotion in the intellectual world, namely: That in the concept of an abstract group mathematics achieved its "last and conclusive creation." And his prediction that "the time of the great mathematicians is past. Our tasks today are those of preserving, rounding off, refining, selection-in place of big dynamic creation, the same clever detailwork which characterized the Alexandrian mathematic of late Hellenism." Could any prophecy have been demonstrated fallacious more quickly and conclusively than this one has been? Also I recall a statement in Struik's little history that "toward the end of the 18th century some of the leading mathematicians expressed the feeling that the field of mathematics was somehow exhausted. The laborious efforts of Euler, Lagrange, D'Alembert, and others had already led to the most important theorems; the great standard texts had placed them, or would soon place them, in their proper setting; the few mathematicians of the next generation would only find minor problems to solve." And this was before 1800 !

If I were given to the vice of prophecy, I would not hestitate to risk my reputation as a prophet with the prediction that mathematics, in this year of our Lord 1952, is only reaching out toward 
maturity, and that given encouragement it will, during the next 50 years, yield new concepts and methods which will revolutionize the subject. I envy those young men who are only on the threshold of their mathematical careers, for they will be possessed of powers that will put their elders to shame. I have already noticed, and no doubt some of you have, evidence of greater powers among recent recruits to the ranks of mathematical research. The future will no doubt see not only an increase in this, but a corresponding improvement will also be noticed in the ranks of undergraduates. I am one of those who believe that genius is not a rare occurrence-genius seems rare chiefly because of lack of opportunity. And in mathematics opportunity is measured to considerable extent by the quantity and magnitude of its concepts and the power of its methods. There is every indication that these are now approaching an all time high. To take a leaf from my own experiences: For some 30 years I have witnessed the growth of topology, sometimes halting, sometimes feverish, from a mere infant to a size where we don't even attempt to give it explicit definition any more. Yet today, I am amazed at the power and ingenuity of the new methods and concepts that are being introduced. And topology is not unique in this.

But it is not only for this reason that I feel as I do about the future of mathematics. I am also a firm believer in the evolutionary character of mathematical development. Concepts are not stable; they continually grow even though their outcroppings are discrete events. This we have noted in the evolution of the concepts of number and curve. And when a concept has evolved so far as to achieve a precise formulation in the mathematical framework then available, its further growth or evolution seems virtually determined by the existence of those logical laws and methods which every mathematician observes and uses. It is much as in the case of a particular axiomatic system, which, once set up, will lead almost inevitably to a certain set of theorems as its logical consequences. Or, to draw once more upon our case history of the concepts of curve and dimension, it is quite clear that the concepts of curve and dimension were already evolving in the mathematical culture stream before being precisely formulated. It is a nice experiment, by the way, to take a group of graduate students who have been trained in basic modern methods, especially in topology, but who have little or no acquaintance with dimension theory, and to give them Poincaré's notions about dimension. If they are any good, they will unfailingly grind out the concept of dimension which corresponds to these notions, just as Poincaré might have if he had had the necessary tools. This would not, of course, be the 
form which Menger and Urysohn gave. Similarly we can find, without reading too much into the prior facts, the evolving forms of concepts such as the calculus and analytic geometry. Their eventual emergence seems to be virtually a certainty. The ways of thought in which we are all trained, and which we all share more or less, seem as much of a guarantee of certain eventual conceptions being synthesized, from given basic material, as do the logical laws for the theorems of an axiomatic system.

As in any evolutionary process, however, the environment cannot be ignored. Just as the individual mathematician does not work in a vacuum, and is influenced by the work of his predecessors and coworkers, so does mathematics itself not evolve in a vacuum. If world crises occur, as we have ample reason to fear they may, they may negate entirely what I said above about the future of mathematical creation. That interplay between evolving concepts and the minds of those individual mathematicians who achieve their syntheses can be interrupted by forces from without. The process of mathematical creation can cease either from stagnation, as from lack of diffusion and the resultant mixing of concepts, or from stifling incidental to major disruptions of society. The former we have no longer any cause to fear; with modern means of communication, and other aids to cultural contacts, our field is more alive than ever before. With good fortune, we may escape the latter, and then I think the prophecy that I said I would make, if I were a prophet, will come true!

\section{BIBLIOGRAPHY}

1. A. N. Whitehead, Science and the modern world, Pelican Mentor Book, N. Y., 1948.

2. H. Poincaré, The foundations of science, Lancaster, Science Press, 1946.

3. J. Hadamard, The psychology of invention in the mathematical field, Princeton University Press, 194.5.

4. O. Veblen, Opening address, Proceedings of the International Congress of Mathematicians, Providence, American Mathematical Society, vol. I, 1952, pp. $124-125$.

5. J. L. Coolidge, A history of geometrical methods, Oxford, 1940.

6. G. Sarton, The study of the history of science, Cambridge, Harvard University Press, 1936.

7. A. Rosenthal, The history of calculus, Amer. Math. Monthly vol. 58 (1951) pp. 75-86.

8. G. Cantor, Gesammelte Abhandlungen, ed. by E. Zermelo, Berlin, Springer, 1932.

9. C. Jordan, Cours d'analyse, Paris, Gauthier-Villars, vol. 1, 1882; vol. 3, 1887.

9a. Second edition of [9], vol. 1, 1893.

10. A. L. Cauchy, Lȩ̧ons sur les applications du calcul infinitesimal à la geometrie, Paris, L'Imprimerie Royale, 1826, vol. 1.

11. L. E. J. Brouwer, Bezweis des Invarianz der geschlossenen Kurve, Math. Ann. vol. 72 (1912) pp. 422-425. 
12. M. Fréchet, Sur quelques points $d u$ calcul fonctionnel, Rend. Circ. Mat. Palermo vol. 22 (1906) pp. 1-74.

13. L. Zoretti and A. Rosenthal, Die Punktmengen, Encyk. d. Math. Wiss., II C 9a, Leipzig, Teubner, 1924.

14. W. Hurewicz and H. Wallman, Dimension theory, Princeton University Press, 1941.

15. K. Menger, Dimensionstheorie, Leipzig, Teubner, 1928.

16. R. L. Wilder, Topology of manifolds, Amer. Math. Soc. Colloquium Publications, vol. 32, New York, 1949.

17. J. M. Child, The early mathematical manuscripts of Leibniz, Chicago, Open Court, 1920.

18. H. Weyl, $A$ half-century of mathematics, Amer. Math. Monthly vol. 58 (1951) pp. 523-553.

19. P. Alexandroff, Dimensionstheorie, Math. Ann. vol. 106 (1932) pp. 161-238.

20. D. R. Curtiss, Fashions in mathematics, Amer. Math. Monthly vol. 44 (1937) pp. 559-566.

UNIVERSITY OF MICHIGAN 